\title{
MICROFABRICATED DRY ADHESIVE DISPLAYING FRICTIONAL ADHESION
}

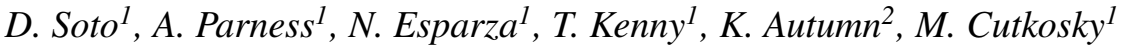 \\ ${ }^{1}$ Stanford University, Stanford, California, USA \\ ${ }^{2}$ Lewis and Clark College, Portland, Oregon, USA
}

\begin{abstract}
Gecko adhesion has inspired many engineered adhesives. Many of these gecko-like synthetic adhesives (GSAs) only replicate the fibrillar aspect of gecko adhesion. We present here a new GSA, MicroWedges, which demonstrates the gecko properties of dynamic adhesion, frictional adhesion, and massive reusability. MicroWedge fibers individually attach and detach during shear sliding, maintaining shear and normal adhesion. MicroWedges also exhibit the gecko-like property of frictional adhesion. Increasing the shear load on a patch of MicroWedges increases the normal adhesion. MicroWedges also demonstrate the gecko-like properties of anisotropy and high $\mu$ '. MicroWedges are fabricated in PDMS from a reusable mold formed from SU-8. The SU-8 is exposed in a dual-side, dual-angle lithography process on transparent wafers.
\end{abstract}

\section{INTRODUCTION}

Biomimetic adhesives inspired by the gecko adhesive system have emerged as a major research field. The gecko adhesive garners this attention since its adhesive system is unlike conventional pressure sensitive adhesives. Conventional adhesive materials are soft, with bulk moduli below $100 \mathrm{kPa}$.[1] The gecko uses micron and nanometer scale hairs of $\beta$-keratin, which has a bulk modulus of $1.6 \mathrm{GPa},[2]$ to form its adhesive. We have taken inspiration from the architectural principles of the gecko's adhesive and previous work with SU-8[3] to create this adhesive. There have been many demonstrations of fibrillar adhesives made from materials that do not have bulk adhesive properties. $[4,5,6,7,8]$ This work builds on previous work by introducing a geometry that allows for frictional adhesion, [9] dynamic adhesion, and excellent longevity. This asymmetrical structure has a sharp angled tip that allows the adhesive to present a variable surface area based on the amount of loading. This allows for adhesive anisotropy, building on the theme of switchability in previous work.[10] The MicroWedges' anisotropic adhesion is described by the frictional adhesion model, which is unlike the familiar embedded friction cone model exhibited by pressure sensitive adhesives such as those on tape or Post-it ${ }^{\circledR}$ Notes (Figure 2).

\section{FABRICATION}

This adhesive is created using a molding process depicted in Figure 3. The reusable mold is created using SU-8 on a quartz wafer with a unique lithography process. We then vacuum cast and cure polydimethylsilox- ane rubber (PDMS) in the mold and peel out to release. To create the mold, we deposit and pattern a thin layer of aluminum on a double polished quartz wafer to create a hardmask. We then spin coat and bake a $200 \mu \mathrm{m}$ layer of SU-8 on top of the aluminum surface. We process the SU-8 according to recommended practice[11]. After SU-8 application, the wafer is placed on a tilted exposure stage. We adjust the stage, correcting for the index of refraction of the SU-8, to achieve the desired angle for the slanted surfaces of the mold. In this work we chose the MicroWedge angle to be $14.8^{\circ}$ giving the wedges a 4:1 aspect ratio. We then expose the wafer to ultraviolet light from the underside. We perform a planar exposure with the second mask in an aligner so that the vertical surfaces are aligned with respect to the slanted surfaces. The SU-8 was then baked and developed using standard processes. With the mold complete, we use it to vacuum cast PDMS. The PDMS was prepared and placed in a vacuum chamber with the mold. The chamber is evacuated and air trapped in the PDMS is liberated. Still under vacuum, the PDMS is poured on the wafer. Air trapped in the mold bubbles through the PDMS and is removed. We then remove the mold from vacuum and spin it to planarize the PDMS backing to the desired thickness. Our backings are in the range of

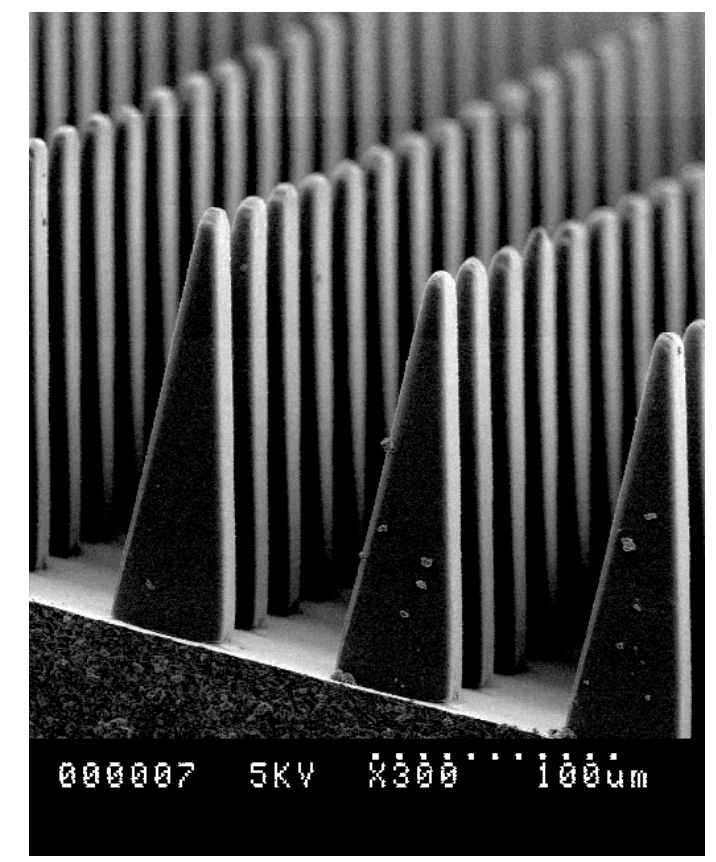

Figure 1: SEM Image of MicroWedges. Structures are $50 \mu \mathrm{m}$ square at the base and are $200 \mu \mathrm{m}$ tall. 
$300 \mu \mathrm{m}$ to $1200 \mu \mathrm{m}$. The PDMS is allowed to cure. The MicroWedges are released by gently peeling the backing layer from the mold.

\section{TESTING}

We test the MicroWedge adhesive on a custom built force testing apparatus with a three-axis motor stage[12] to control friction and loading at arbitrary angles (Figure 4). A MicroWedge patch of $\sim 1 \mathrm{~cm}^{2}$ is affixed by a conventional tape adhesive to a fixture on a load cell. The fixture sits atop a two-axis tilt stage that provides for the sample to be coplanar with the glass test surface. The glass stage is lowered and brought into contact with the adhesive. After initial contact, the glass is lowered further, compressing the sample. This compression phase is referred to as the preload. At this point, the stage is moved at an angle to the sample. If the sample is moved at an angle normal to the sample, it is called a load-pull (LP) test. In the case of the stage moving parallel and shearing the adhesive, the test is called a load-drag-pull (LDP) test. Intermediate angles are also tested. We collect data for both shear force and normal force adhesion during the period of contact. We also record the forces at the moment that the adhesive breaks contact by either detachment or slipping.

\section{RESULTS}

Dynamic Adhesion

The force trace plot (Figure 5) allows us to see the forces exerted by the adhesive as a function of time. The plot shows that dynamic sliding adhesion is obtained where despite slipping, both shear and normal adhesion forces are exerted. The adhesive is able to simultaneously maintain $4 \mathrm{kPa}$ of adhesion and $20 \mathrm{kPa}$ of shear. This implies that individual hairs that have detached are able to reattach and exert adhesive force. This property

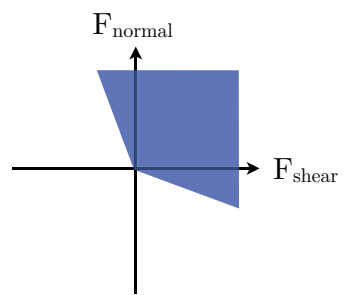

Frictional Adhesion

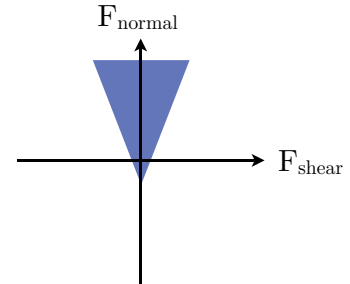

Embedded Cone
Figure 2: Comparison of Frictional Adhesion and Embedded Cone models. The shaded areas represent regions of stability. The unshaded areas represent areas where the adhesive will pull away or slip relative to the substrate. The frictional adhesion model is unique as increasing the shear on the adhesive generates more adhesion. Another unique feature is that the adhesion vanishes when the shear is removed.

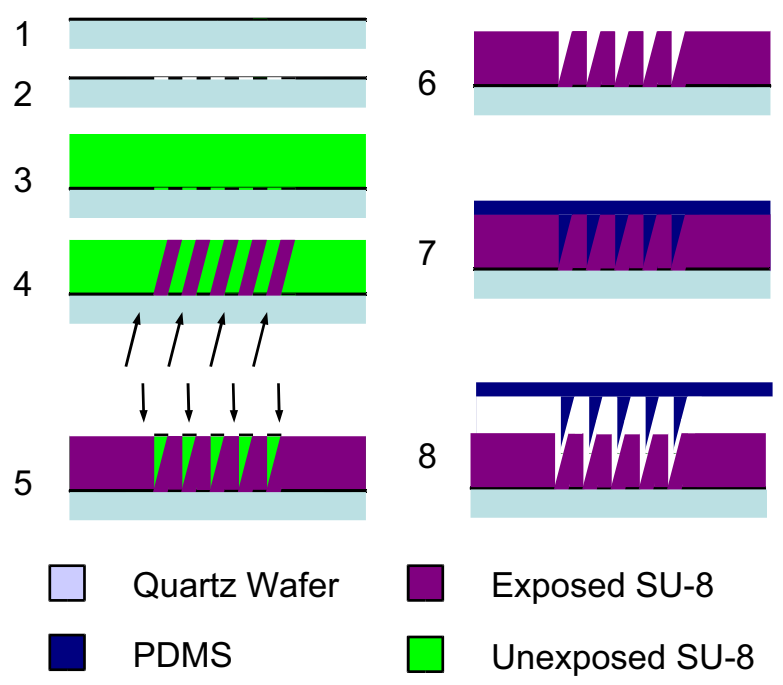

Figure 3: Fabrication Sequence 1) Deposit aluminum on quartz wafer 2) Pattern aluminum to create self-aligned mask 3) Deposit $S U-8$ on top of aluminum 4) Angled self-aligned UV exposure from backside 5) Align mask to topside and UV expose 6) Develop 7) Cast and spin PDMS 8) Peel out cast MicroWedges and backing layer

allows the MicroWedge adhesive a graceful failure mode desirable for robotics or locomotion applications. Other GSAs have demonstrated similar results using a spherical surface with a radius of $\sim 5 \mathrm{~mm}$. [8] These tests don't necessarily demonstrate the reattachment of fibrils since only a portion of the sample's fibers are in contact.

\section{Frictional Adhesion}

The limit surface in Figure 6, obtained through many trials over a range of preload angles, preload depths, and exit angles, is a map of the release points for different

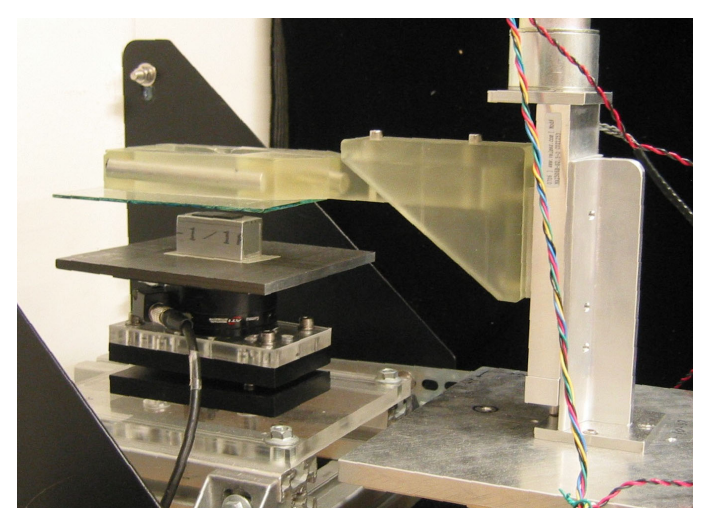

Figure 4: Force measurement apparatus. Photo shows MicroWedge adhesive samples on mounting plate affixed to load cell. Load cell is above two-axis tilt plate for plane-plane alignment. The glass plate (not in contact) is above the sample. The motorized stages are seen on the right side of the photo. 
shear to adhesion ratios. The curve creates a limit surface of conditions where the adhesive contact will fail, either by shear or normal movement. The limit surface shows that as greater shear is exerted, the adhesion increases. The limit surface of the flat control in Figure 7, shows behavior similar to the embedded cone model where increasing shear decreases the available normal force. Also, the limit surface of the flat control does not pass through the origin. This implies that there must be a normal force exerted at detachment when there is no shear.

\section{Longevity}

We have performed testing of the adhesive over thousands of LDP cycles. When loaded to a constant displacement depth, the MicroWedges retain $50 \%$ of their adhesion and $86 \%$ of their shear over the 30,000 trials. This is in contrast to conventional pressure sensitive adhesives as well as other synthetic fibrillar adhesives which degrade more quickly with repeated use. Each of these trials was a $10 \mathrm{~mm}$ drag resulting in a total dragged distance of $300 \mathrm{~m}$.

\section{DISCUSSION}

We believe that this adhesive is an important advancement in applications where shear force, anisotropy, and reusability are key properties. These properties are especially important for climbing applications. Traditional pressure sensitive adhesives are not suitable for climbing since they require large forces of detachment. This adhesive exhibits frictional adhesion and as can be seen in Figures 2 and 6, when the shear force is removed, the normal adhesion vanishes, allowing effortless detachment. This ease of detachment enables climbing stability. Another key property for climbing applications is the dynamic adhesion. The MicroWedge adhesive is able to maintain adhesion while sliding. This prevents

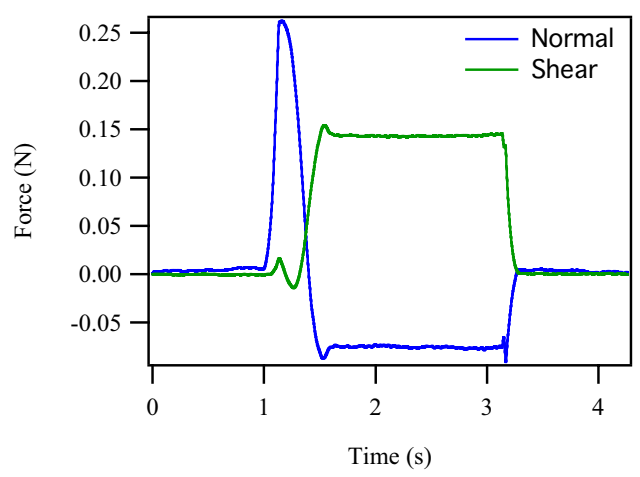

Figure 5: MicroWedge Force Trace. The normal trace shows the initial compression phase followed by a region of adhesion during the parallel drag. In the shear trace, we see that there is a shear force created by the adhesive at the same time as the normal adhesion force.

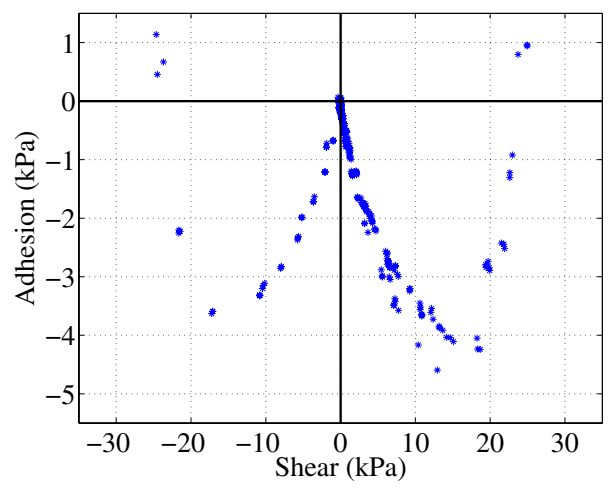

Figure 6: Frictional Adhesion Plot for $50 \mu \mathrm{m}$ Diameter MicroWedges. We see that the adhesion is increased as the shear increases. Also, if the shear is decreased to zero, the adhesion vanishes and the adhesive can be removed with no force.

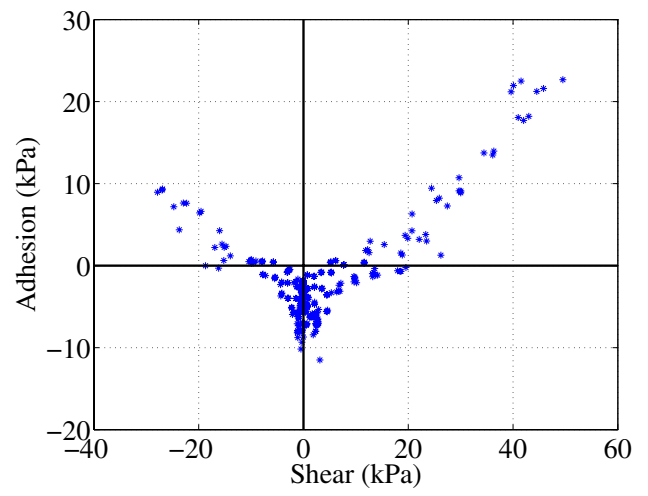

Figure 7: Frictional Adhesion Plot for PDMS Flat Control Sample. The limit surface does not intersect the origin and increasing shear leads to diminished adhesive capability.

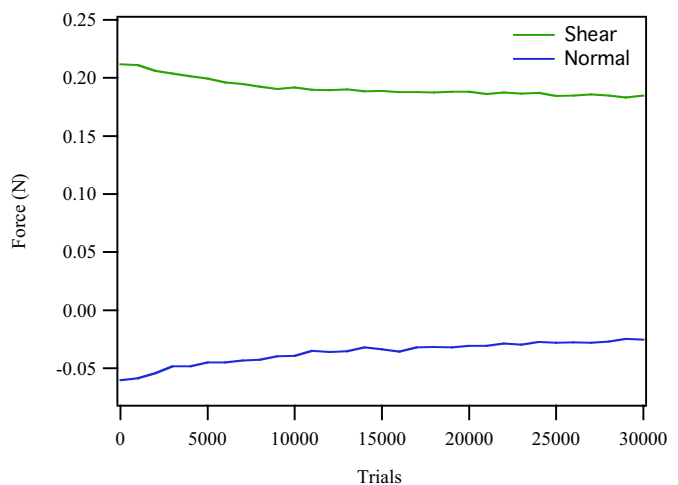

Figure 8: Lifetime Test of MicroWedge Adhesive. 30,000 trials performed of 10mm drag. Adhesion fell to $50 \%$ of initial and shear fell to $86 \%$ of initial during test. This longevity is superior to other pressure sensitive adhesives. 
catastrophic failures of adhesion. For a climbing robot, graceful failures in adhesion leading to sliding are preferable to complete detachment.

\section{CONCLUSION}

A novel geometry for a GSA has been fabricated and tested. The MicroWedge adhesive displays increasing adhesion with increasing shear, the ability to maintain adhesion while sliding, and maintains $56 \%$ of its initial adhesion after 30,000 cycles. Future work will investigate the effect of material properties and device geometry on performance.

\section{ACKNOWLEDGEMENTS}

Work was performed in part at the Stanford Nanofabrication Facility (a member of the National Nanotechnology Infrastructure Network) which is supported by the National Science Foundation under Grant ECS-9731293, its lab members, and the industrial members of the Stanford Center for Integrated Systems. This work was supported by the CIS New User Grant Program at the Stanford Nanofabrication Facility.

\section{REFERENCES}

[1] C. Gay and L. Leibler, "Theory of Tackiness", Physical Review Letters, (1999).

[2] A. Peattie, C. Majidi, A. Corder, and R.J. Full, "Ancestrally high elastic modulus of gecko setal $\beta$ keratin", The Journal of the Royal Society Interface, 4, (2007).

[3] H. Sato, Y. Houshi, and S. Shoji, "Three-dimensional micro-structures consisting of high aspect ratio inclined micro-pillars fabricated by simple photolithography", Microsystem Technologies, 10, (2004).

[4] A. Geim, S. Dubonos, I. Grigorieva, K. Novoselov, A.A. Zhukov, and S.Y. Shapoval, "Microfabricated adhesive mimicking gecko foot-hair", Nature Materials, 2, (2003).
[5] S. Gorb, M. Varenberg, A. Peressadko, and J. Tuma, "Biomimetic mushroom-shaped fibrillar adhesive microstructure", Journal of The Royal Society Interface, 4, (2007).

[6] L. Ge, S. Sethi, L. Ci, P. Ajayan, and A. Dhinojwala, "Carbon nanotube-based synthetic gecko tapes", Proceedings of the National Academy of Sciences, 104, 26, (2007)

[7] B. Schubert, J. Lee, C. Majidi, and R.S. Fearing, "Sliding-induced adhesion of stiff polymer microfibre arrays. II. Microscale behaviour" Journal of The Royal Society Interface (2008)

[8] M.P. Murphy, B. Aksak, M. Sitti "Adhesion and anisotropic friction enhancements of angled heterogeneous micro-fiber arrays with spherical and spatula tips", Journal of Adhesion Science and Technology, 21, (2007)

[9] K. Autumn, A. Dittmore, D. Santos, M. Spenko, and M. Cutkosky, "Frictional adhesion: a new angle on gecko attachment", The Journal of Experimental Biology, 209, (2006).

[10] M. T. Northen, K.L. Turner, C. Greiner, and E. Arzt, "A hierarchical gecko-inspired switchable adhesive", Technical Digest of the 2006 Solid-State Sensor and Actuator Workshop, Hilton Head Isl., SC, 2006, Transducer Research Foundation, Cleveland (2006), pp. 43-46.

[11] http://www.microchem.com

[12] D. Santos, M. Spenko, A. Parness, S. Kim, and M. Cutkosky, "Directional adhesion for climbing: theoretical and practical considerations", Journal of Adhesion Science and Technology, 21, (2007) 